\title{
More than meets the eye: Monocular syphilis in an HIV-negative patient
}

\author{
Amanda L. Strickland ${ }^{1}$, Nicole E. Strickland ${ }^{1}$, Mark Feldman ${ }^{2}$ \\ 1. Department of Internal Medicine, University of Texas Southwestern Medical Center, Dallas, TX, USA. 2. Department of \\ Internal Medicine, Texas Health Presbyterian Hospital of Dallas, Dallas, TX, USA. \\ Correspondence: Mark Feldman. Address: 8200 Walnut Hill Lane, Dallas, Texas 75232, USA. Email: \\ markfeldman@texashealth.org
}

Received: February 8, 2014

DOI : $10.5430 /$ crim.v1n2p29

Accepted: March 4, $2014 \quad$ Online Published: March 14, 2014

URL: http://dx.doi.org/10.5430/crim.v1n2p29

\begin{abstract}
A 50-year old heterosexual male presented with discomfort in his left eye and abnormal behavior. Physical examination showed a red eye suggestive of uveitis. He was found to have a reactive rapid plasma reagin (RPR) test in high titer and a cerebrospinal fluid (CSF) pleocytosis, with a reactive Venereal Disease Research Laboratory (VDRL) titer in the CSF. Testing for human immunodeficiency virus (HIV)-1 and HIV-2 was negative. He was diagnosed with ocular/neurosyphilis and treated with intravenous (IV) penicillin G for 14 days followed by an intramuscular injection of benzathine penicillin, with complete resolution of ocular findings and behavioral changes. This report illustrates an unusual presentation of ocular/neurosyphilis in an HIV-negative individual with no other presenting symptoms and the efficacy of antibiotic therapy for this condition. Cases of syphilitic uveitis in HIV-negative patients in the literature are reviewed.
\end{abstract}

\section{I ntroduction}

The incidence of neurosyphilis, an infection of the central nervous system by the spirochete Treponema pallidum, has declined since the introduction of antibiotics. Nowadays, the vast majority of patients who have neurosyphilis are HIV-positive. We present the case of an HIV-negative individual in whom monocular involvement (uveitis) and behavioral changes were the only presenting signs and symptoms of neurosyphilis.

\section{Case presentation}

A 50-year-old Caucasian male complained of scratchiness in his left eye, along with some clear weeping discharge, photosensitivity and blurry vision. These symptoms had started approximately two weeks earlier. He denied currently or ever having any genital sores/chancres, rash, urethral discharge, dysuria, fever, headache, or other neurological symptoms.

He had initially presented with symptoms compatible with bacterial conjunctivitis and was prescribed topical gentamicin. A week later his ocular symptoms had not resolved and he was referred to an ophthalmologist, who diagnosed pan-uveitis and performed an anterior chamber paracentesis. The ophthalmologist injected foscarnet and ganciclovir into the eye and also prescribed oral valacyclovir and trimethoprim/sulfamethoxazole for empiric coverage of infections with 
cytomegalovirus (CMV), herpes simplex virus (HSV), and Toxoplasma gandii. Atropine and prednisolone ophthalmic drops were also prescribed.

His past medical and surgical history was significant for diet-controlled pre-diabetes, erectile dysfunction, gonorrhea, intravenous drug abuse, and gallstones treated by cholecystectomy. He was an active smoker of cigarettes and cigars. He used to drink two six-packs of ale daily for 15 years. He reported being heterosexual with multiple sexual partners. His only other oral medication beside the two antibiotics was sildenafil.

Physical examination revealed an anxious but alert and oriented Caucasian male complaining of significant left eye discomfort. His temperature was $36.3^{\circ} \mathrm{C}$, blood pressure $128 / 73 \mathrm{mmHg}$, and BMI $25.4 \mathrm{~kg} / \mathrm{m}^{2}$. Oxygen saturation was $98 \%$ on room air. The patient had intact extraocular muscles and normal visual acuity. His right eye was clear and unremarkable, but the left eye was erythematous. There was anisocoria (left pupil $6 \mathrm{~mm}$ and right pupil $4 \mathrm{~mm}$ ), perhaps related to recent use of atropine drops in the left eye. Cardiovascular, pulmonary, gastrointestinal, musculoskeletal and genital examinations were normal. Cranial nerves were intact. There were no focal sensory or motor deficits, dysmetria, or dyskinesia and the Romberg sign was absent. The patient had a normal gait, though he tended to pace in circles alternating his weight on each foot. He had difficulty sitting still or standing still when unprovoked. He exhibited an anxious and agitated affect, made many inappropriate, hypersexual comments, and was combative. Skin exam was hindered by multiple tattoos, but no rashes or lesions were seen.

A complete blood count and comprehensive metabolic panel were normal. A RPR blood test was reactive at a titer of 1:64, and an antibody test to Treponema pallidum was reactive. Testing for HIV-1 and HIV-2 antibodies and p24 antigen was negative. Polymerase chain reaction (PCR) testing of the ocular fluid was negative for HSV, varicella zoster virus, and Toxoplasma gandii.

CSF examination revealed a pinkish fluid with 64,000 and 68,000 red blood cells/ $\mathrm{mm}^{3}$ in tubes 1 and 3, with no xanthochromia. The CSF glucose concentration was normal $(68 \mathrm{mg} / \mathrm{dl})$ but the protein content was quite elevated (187 $\mathrm{mg} / \mathrm{dl}$ ). The CSF white blood cell (WBC) count was elevated (294 and 313/ $\mathrm{mm}^{3}$ in tubes 1 and 3), with the majority of the leucocytes being lymphocytes or monocytes. The VDRL in the CSF was reactive (titer, 1:4). HSV PCR of the CSF was negative.

He received a 14 day course of treatment of 4 million units IV penicillin G every 4 hours, followed by a final intramuscular injection of 2.4 million units of benzathine penicillin. Notably, as he received his treatment the patient's behavior became calmer and he was no longer anxious. His ocular symptoms and signs resolved. The patient was discharged in good condition and instructed to follow up with the ophthalmologist and his local health department, although the patient has not followed up with any health department in the state for re-evaluation.

\section{Discussion}

Ocular syphilis, caused by the spirochete Treponema pallidum, is an uncommon manifestation of secondary or tertiary syphilis and can involve most structures of the eye ${ }^{[1]}$. Manifestations of ocular syphilis include granulomatous and non-granulomatous anterior, posterior or pan-uveitis, vitritis, interstitial keratitis, chorioretinitis, neuroretinitis, retinal vasculitis, and papillitis. Since syphilis is not uncommon among HIV-positive patients, clinical suspicion for syphilitic involvement of the eye should remain high in any known HIV-positive patient presenting with any eye symptoms and/or signs ${ }^{[1,2]}$.

Recent single case reports (including this report) and small case series ${ }^{[3-9]}$ illustrate the need to extend this clinical suspicion to HIV-negative patients, especially those with a promiscuous sexual history. Increasing rates of syphilis in the United Kingdom (UK), United States (US) and elsewhere have brought to attention cases of ocular syphilis in 
HIV-negative, heterosexual patients. Although some of these affected patients presented with a generalized papulosquamous rash, a recent case series from China reported 8 HIV-negative patients with ocular uveitis as their only initial presentation of syphilis ${ }^{[6]}$. Our patient was treated for bacterial conjunctivitis, CMV infection, HSV infection and toxoplasmosis before the correct diagnosis of monocular syphilitic pan-uveitis was made.

Diagnosis of ocular syphilis can be confirmed with a syphilis serologic test. Vitreous PCR for Treponema pallidum, a useful adjunctive diagnostic test ${ }^{[2,3,7]}$, was not performed in our patient due to the low volume of vitreous sample. Unless signs of concurrent neurosyphilis are present (e.g., headache, focal neurological signs), performance of CSF studies in ocular syphilis is controversial, since results may not change treatment options and since negative CSF results, as seen in case reports, can perpetuate diagnostic uncertainty and delay adequate treatment ${ }^{[1,10,11]}$. Treatment of ocular syphilis is the same neurosyphilis regimen of 24 million units of IV penicillin G daily for 10-14 days ${ }^{[12]}$. However, not all cases of ocular syphilis are fully cured with this treatment, and relapses can occur even in immunocompetent patients receiving proper treatment.

The previous case reports and small case series of patients with syphilitic uveitis, with or without other manifestations of neurosyphilis, in HIV-negative individuals are summarized in Table 1. The largest case series is from Hong et al in China who collected 8 cases over a 13-year period ${ }^{[6]}$. Most of the other cases were reported in the United Kingdom ${ }^{[3,8,9]}$. HIV-negative patients with syphilitic uveitis ranged in age from 30 to 80 years (mean, 54 years) and the majority of them (78\%) were men. Uveitis was unilateral, as in our patient, in approximately half of the cases and bilateral in the other half. A generalized rash was present in only 4 of 19 cases (21\%); 2 cases from Mexico, like ours, had a positive CSF VDRL titer.

Table 1. Published cases of ocular syphilis with uveitis in HIV-negative patients

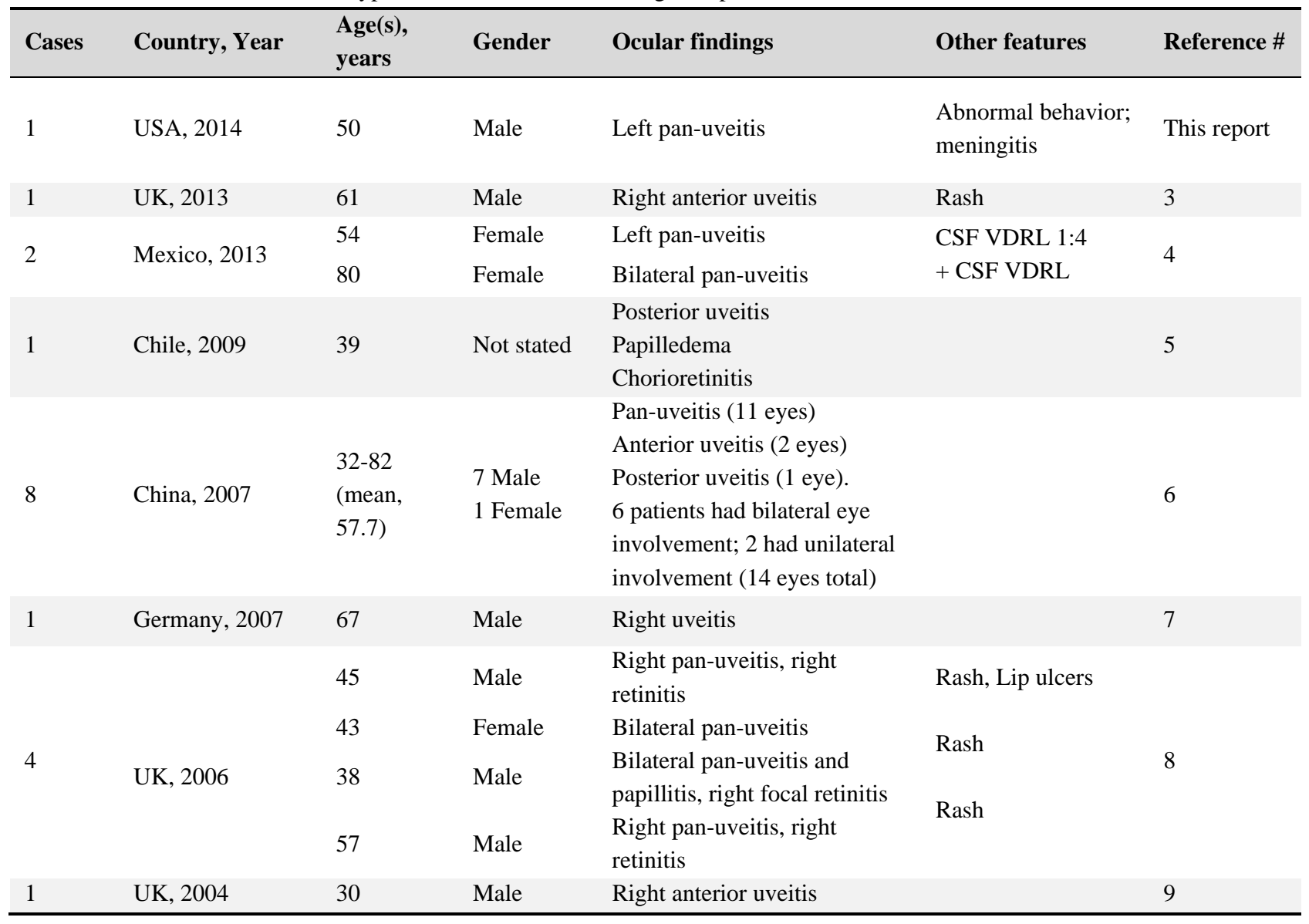


The Centers for Disease Control and Prevention (CDC) recommends repeat CSF examinations at 6 months and twelve months following treatment of neurosyphilis to determine changes in CSF VDRL or protein levels. They recommend more frequent re-evaluations if follow-up is uncertain. Checking the CSF WBC count is considered a more sensitive measure of therapy effectiveness, because the CSF VDRL titers and protein levels tend to decline more slowly than the CSF WBC count. CSF re-evaluation should continue every 6 months until the WBC counts or protein levels become normal; if they do not, or if non-treponemal test titers do not decline fourfold within 6 to twelve months, retreatment should be considered due to the possibility of treatment failure or reinfection.

\section{Acknowledgments}

Edward L. Goodman. M.D. kindly reviewed the manuscript.

\section{References}

[1] Margo CE, Hamed LM. Ocular syphilis. Surv Ophthalmol. 1992; 37(3): 203-20. http://dx.doi.org/10.1016/0039-6257(92)90138-J

[2] Booth J, Rodger A, Singh J, et al. Syphilitic panuveitis with retinal necrosis in an HIV positive man confirmed by Treponema pallidum PCR. J Infect. 2009; 59(5): 373-5. http://dx.doi.org/10.1016/j.jinf.2009.08.022

[3] Troutbeck R, Chhabra R., Jones NP. Polymerase chain reaction testing of vitreous in atypical ocular syphilis. Ocul Immunol Inflamm. 2013; 21(3): 227-30. http://dx.doi.org/10.3109/09273948.2013.770887

[4] Gonzalez-Duarte A, Lopez ZM. Neurological findings in early syphilis: a comparison between HIV positive and negative patients. Neurol Int. 2013; 5(4): e19. http://dx.doi.org/10.4081/ni.2013.e19

[5] Lasso MB, Balcells EM, Fernandez AS, et al. Neurosyphilis in the patients with and without HIV infection: description and comparison of two historical cohorts. Rev Chil Infect. 2009; 26(6): 540-7.

[6] Hong M-C, Sheu S-J, Wu T-T,et al. Ocular uveitis as the initial presentation of syphilis. J Chin Med Assoc. 2007; 70(7): $274-280$. http://dx.doi.org/10.1016/S1726-4901(07)70004-7

[7] Müller M, Ewert I, Hansmann F, et al. Detection of Treponema pallidum in the vitreous by PCR. Br J Ophthalmol 2007; 91(5): 592-5. http://dx.doi.org/10.1136/bjo.2006.110288

[8] Doris JP, Saha K, Jones NP et al. Ocular syphilis: the new epidemic. Eye. 2006; 20(6): 703-5. http://dx.doi.org/10.1038/sj.eye.6701954

[9] Durnian JM, Naylor G, Saeed AM. Ocular syphilis: the return of an old acquaintance. Eye. 2004; 18(4): 440-2. http://dx.doi.org/10.1038/sj.eye.6700687

[10] Browning DJ. Posterior segment manifestations of active ocular syphilis, their response to a neurosyphilis regimen of penicillin therapy, and the influence of human immunodeficiency virus status on response. Ophthalmology 2000; 107(11): $2015-23$.

[11] Workowski KA., Berman S. Sexually transmitted diseases treatment guidelines, 2010. MMWR Recomm Rep 2010; 59(RR-12): 1-110. 